\title{
Videoanálise do voo de um fidget spinner: torque e momento angular
}

\author{
The video analysis of a fidget spinner flight: torque and angular momentum
}

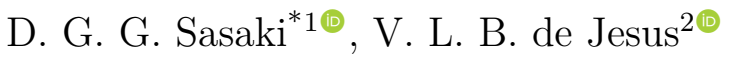 \\ ${ }^{1}$ Centro Federal Educação Tecnológica Celso Suckow da Fonseca, Maracanã, RJ, Brasil \\ ${ }^{2}$ Instituto Federal de Educação, Ciência e Tecnologia do Rio de Janeiro, campus Nilópolis, RJ, Brasil
}

Recebido em 22 de Agosto de 2019. Aceito em 04 de Outubro de 2019

\begin{abstract}
Poderia o mundialmente famoso brinquedo fidget spinner ser usado em sala de aula para ilustrar os conceitos de momento de inércia, torque e momento angular? Curiosamente, foram raros os artigos de ensino relacionados ao tema que foram publicados até a presente data. Neste trabalho apresentamos uma situação real composta por duas configurações experimentais extremamente simples de lançamento oblíquo de um spinner com e sem rotação inicial do tipo spin. Definimos por rotação do tipo spin como qualquer rotação em que o spinner gira em torno de um de seus eixos principais de simetria. A técnica escolhida para coleta dos dados foi a videoanálise, usando o software gratuito Tracker. Os principais resultados foram (i) Apresentar um experimento de execução rápida e fácil e que aborde de forma simultânea os conceitos de torque, momento angular orbital e momento angular de spin. (ii) Analisar de forma qualitativa as semelhanças e as diferenças entre as orientações dos momentos angulares orbitais e de spin nos dois tipos de lançamento oblíquo do spinner (iii) Obter uma estimativa quantitativa das magnitudes dos momentos angulares de spin em relação ao respectivo eixo principal de simetria do spinner, em ambos os casos.
\end{abstract}

Palavras-chave: ensino de física, videoanálise, torque, momento angular, spinner.

Could the world-famous fidget spinner toy be used in the classroom to illustrate the concepts of moment of inertia, torque and angular momentum? Curiously, there are very few papers related to the topic that have been published so far. In this work, we present a real situation consisting of two extremely simple experimental configurations of oblique launching of a spinner with and without initial spin type rotation. We define spin type rotation as any rotation in which the spinner rotates around one of its main symmetry axes. The technique chosen for data collection was the video analysis, using the free software Tracker. The main results were (i) To present a fast and easy execution experiment that simultaneously approaches the concepts of torque, orbital angular momentum and spin angular momentum. (ii) Analyze in a qualitative way the similarities and differences between the orientations of the orbital and spin angular momenta for two types of oblique launching (iii) Obtain a quantitative estimative of the magnitudes of the spin angular momenta in relation to the respective symmetry axis of the spinner, in both cases.

Keywords: physics teaching, video analysis, torque, angular momentum, fidget spinner.

\section{Introdução}

Em 2017, um brinquedo chamado de fidget spinner se tornou extremamente famoso entre crianças, adolescentes e até mesmo adultos, espalhados pelo mundo inteiro. Por conta dessa súbita popularidade, uma questão natural emerge: o spinner poderia ser usado para ilustrar conceitos de física, tais como momento de inércia, torque e momento angular? Curiosamente, foram raros os artigos de ensino relacionados ao tema que foram publicados até a presente data [1-4]. Esse descompasso entre o enorme interesse pelo brinquedo entre os jovens e os trabalhos que abordam o seu possível uso em sala de aula revela a grande inércia dos sistemas de educação para entrar em

*Endereço de correspondência: vitor.jesus@ifrj.edu.br sintonia e acompanhar a intensa dinâmica dos assuntos que mobilizam a juventude no mundo atual.

Com o intuito de preencher essa lacuna, este artigo apresenta uma configuração experimental simples e fácil de lançamento oblíquo de um spinner com e sem rotação inicial do tipo spin, com o intuito de discutir conceitos como torque, momento angular orbital e momento angular de spin. Definimos por rotação do tipo spin como qualquer rotação em que o spinner gira em torno de um de seus eixos principais de simetria. Naturalmente, a forma mais comum de rotação do tipo spin para qual o uso do brinquedo foi projetado é aquela em torno do seu eixo principal de simetria que é equidistante dos seus rolamentos. Denominamos essa modalidade de rotação natural de spin e identificamos o seu eixo com a direção 
z' de um sistema de coordenadas solidário ao centro de massa (CM) do spinner.

A técnica escolhida para coleta dos dados foi a videoanálise, usando o software gratuito Tracker [5].

\section{O experimento}

As filmagens dos lançamentos oblíquos do spinner foram feitas utilizando a opção slow motion (120 fps) da câmera

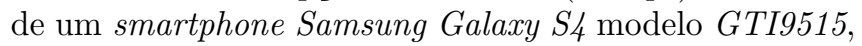
e a videoanálise foi realizada utilizando o software livre Tracker. O smartphone foi posicionado a aproximadamente $1 \mathrm{~m}$ do plano onde ocorreu o lançamento oblíquo do spinner. Os dados foram obtidos marcando-se os pontos no vídeo manualmente, evitando-se a opção autotracker.

Foram analisados dois lançamentos oblíquos, o primeiro sem e o segundo com rotação inicial de spin. Os lançamentos foram realizados com a ajuda de uma "catapulta", cujo papel foi desempenhado pela tampa da parte superior de uma cafeteira italiana. Dois livros foram utilizados como "niveladores" mantendo assim o plano do spinner na horizontal antes do lançamento. Ao se abrir a tampa da cafeteira com um gesto rápido dos dedos, mantendo o bojo preso à mão, o spinner é lançado obliquamente.

Deve-se tomar cuidado com o posicionamento do spinner sobre a tampa para assegurar que a trajetória do seu $\mathrm{CM}$, após o lançamento, esteja em um plano perpendicular ao eixo de rotação da tampa da cafeteira e, portanto, paralelo ao plano de filmagem da câmera. As trajetórias do spinner, sem e com rotação natural de spin, podem ser claramente visualizadas por meio de fotografias estroboscópicas, ilustradas nas Figs. 1 e 2, respectivamente. Nas Figs. 1 e 2, o eixo $\mathbf{z}$ aponta para cima, o eixo $\mathbf{x}$ para a direita e o eixo y para dentro das figuras. No instante inicial, antes do lançamento, quando o spinner ainda se encontra em sobre a tampa horizontal, o sistema de coordenadas inercial xyz coincide com o sistema de

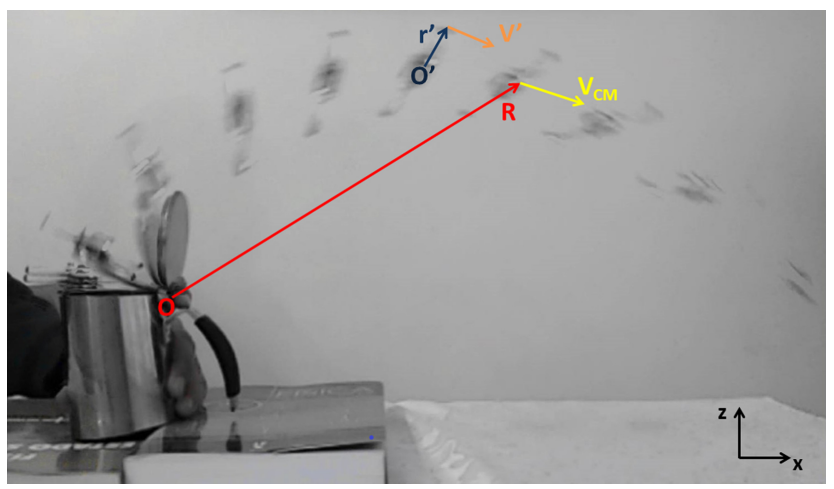

Figura 1: Foto estroboscópica do movimento do lançamento oblíquo do spinner sem rotação natural de spin. Em cores, estão representados os vetores relevantes para a descrição do movimento. No canto inferior direito, foi inserido um sistema de coordenadas inercial usado como referência.

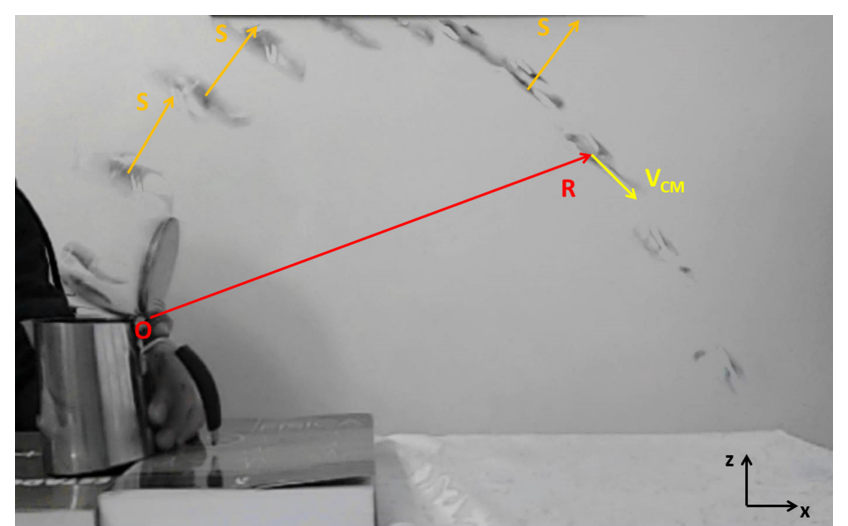

Figura 2: Foto estroboscópica do movimento do lançamento oblíquo do spinner com rotação natural de spin. Em cores, estão representados os vetores relevantes para a descrição do movimento. No canto inferior direito, foi inserido um sistema de coordenadas inercial usado como referência.

coordenadas não inercial x'y'z', localizado no CM do spinner.

A mera inspeção visual das fotografias já é bastante reveladora da diferença entre o comportamento do spinner quando é lançado, sem e com rotação natural de spin. De fato, na Fig. 1 percebe-se que o spinner inicialmente em repouso, sem qualquer tipo de spin, adquire durante o lançamento um movimento de rotação em torno de um eixo perpendicular à fotografia, no sentido horário.

Por outro lado, a Fig. 2 mostra que o lançamento do spinner já animado com uma rotação natural de spin é bem distinta, pois o spinner mantém uma orientação espacial fixa ao longo de todo o seu voo, após perder o contato com a tampa da cafeteira e, portanto, chega ao final do movimento ainda de "cabeça para cima", apenas um pouco inclinado em relação à sua posição original.

Essa discrepância da orientação espacial do spinner durante o voo nos dois casos é bastante curiosa e costuma ser imprevisível para a maioria dos estudantes tanto de ensino médio quanto de nível superior e por isso tem grande potencial de despertar a curiosidade dos alunos e incentivar o seu interesse no tema.

\subsection{Análise qualitativa das imagens}

Tomando como referência um sistema de coordenadas inercial com origem fixa $\boldsymbol{O}$ sobre o eixo de rotação da tampa da cafeteira, podemos afirmar que o momento angular total do spinner $(\vec{J})$ é a soma vetorial do seu momento angular orbital $(\vec{L})$ com o seu momento angular de spin $(\vec{S})[6]$. Entende-se por momento angular orbital, aquele proveniente do movimento do CM do spinner, em relação ao ponto $\boldsymbol{O}$. Por sua vez, definimos o momento angular de spin como a resultante do momento angular de todos os pontos de massa do spinner, em relação ao seu CM (ponto O'). Nos dois casos específicos do experimento, o momento angular de spin vai corresponder 
a um vetor na direção de um dos eixos principais de simetria (eixo z') do spinner.

Apesar de sua simplicidade de execução e visualização, esse experimento proporciona uma série de perguntas qualitativas instigantes e relacionadas que podem ser debatidas em sala de aula, a saber:

1. Qual a direção e sentido dos momentos angulares orbital, de spin e total do spinner, durante o seu voo, em cada caso?

2. Qual a direção e sentido das forças que atuam no spinner durante o movimento da tampa da cafeteira? Quais delas exerce torque sobre o spinner?

3. Qual a direção e sentido do torque resultante aplicado pela tampa da cafeteira em relação a um ponto localizado no eixo de rotação da tampa, em ambos os casos?

4. Por que a orientação espacial do spinner durante o voo no caso 2 é tão distinta do caso 1 ?

\subsubsection{Caso 1: Lançamento do spinner sem rotação natural de spin}

No instante inicial, o momento angular de spin $(\vec{S})$ e o momento angular orbital $(\vec{L})$ são nulos e, consequentemente, o momento angular total $(\vec{J}=\vec{L}+\vec{S}=0)$. Quando a tampa entra em movimento, o spinner adquire simultaneamente momento angular orbital e de spin. Durante esse período, o spinner está sujeito a três forças: peso, normal e atrito. Após perder o contato com a tampa e iniciar o seu voo, o spinner fica sob a ação exclusiva da força peso e da força de resistência do ar, sendo essa última desconsiderada em nosso modelo.

Na Fig. 1 também estão desenhados, em um dado ponto da trajetória, o vetor velocidade do $\mathrm{CM}$ do spinner $\left(\vec{V}_{C M}\right)$ e o seu vetor posição $(\vec{R})$, ambos medidos em relação à origem $\boldsymbol{O}$, bem como o vetor posição $\left(\overrightarrow{r^{\prime}}\right)$ de um ponto da borda do spinner e a sua respectiva velocidade vetorial $\left(\overrightarrow{v^{\prime}}\right)$, ambos em relação ao CM. Nessa situação, os momentos angulares orbital $(\vec{L})$ e de $\operatorname{spin}(\vec{S})$ podem ser calculados pelas seguintes expressões $[6]$ :

$$
\begin{aligned}
& \vec{L}(t)=\vec{R}(t) \times M \vec{V}_{C M}(t) \\
& \vec{S}=\sum_{i}^{N} \vec{S}_{i}=\sum_{i}^{N} \vec{r}_{i}^{\prime} \times m_{i} \vec{v}_{i}^{\prime}
\end{aligned}
$$

Durante o voo, somente a força peso atua, logo o seu torque em relação ao CM é nulo, acarretando a conservação do momento angular de $\operatorname{spin}(\vec{S})$. Como a trajetória do CM do spinner é uma parábola contida no plano da figura, a direção e o sentido do momento angular orbital $(\vec{L})$, medido em relação à origem $\boldsymbol{O}$, são fixos. Porém, o seu o módulo, varia e, portanto, é um vetor dependente do tempo. A variação do momento angular orbital durante o voo é atribuída ao torque não nulo da força peso, quando medido em relação à origem $\boldsymbol{O}$.
A despeito do fato de que o vetor momento angular de spin é constante e o módulo do momento angular orbital varia com tempo, ambos os sentidos desses vetores durante o voo do spinner, obtidos pela regra da mão direita, apontam para dentro figura (sentido do eixo y). Logo, o momento angular total $(\vec{J})$ também está nessa direção e sentido.

Por fim, pode-se concluir que por conta da conservação do momento angular de spin, o spinner gira em torno do eixo y com velocidade angular de rotação constante.

Qual seria a causa do torque que originou o momento angular total? Enquanto o spinner está em contato com a tampa, a força de atrito não exerce torque porque atua na mesma direção do vetor posição, tomado em relação à origem $\boldsymbol{O}$. Pelo mesmo motivo, a componente da força peso paralela ao plano da tampa também não provoca torque. Portanto, apenas a força normal e a componente da força peso ortogonal à tampa efetuam torque sobre o spinner. Esses torques, embora opostos, não se cancelam, pois o módulo da força normal supera a intensidade da componente da força peso no sentido oposto, já que o $\mathrm{CM}$ adquire aceleração. Consequentemente, pela regra da mão direita, o torque resultante sobre o spinner está na direção ortogonal ao plano da figura e o sentido para dentro (sentido y).

\subsubsection{Caso 2: Lançamento do spinner com rotação natural de spin}

Naturalmente, as forças que atuam sobre o spinner quando ainda em contato com a tampa da cafeteira, assim como ao longo do seu voo são as mesmas do caso anterior. Além disso, pelo mesmo motivo, a componente da força peso paralela à tampa e a força de atrito não exercem torque sobre o spinner, em relação ao ponto $\boldsymbol{O}$.

Dessa vez, no instante inicial, o momento angular orbital ainda é nulo, mas o momento angular de spin é não nulo e aponta no sentido do eixo z. Quando a tampa entra em movimento, o spinner adquire momento angular orbital, que aponta no sentido y, como no caso 1. Porém, o momento angular de spin vai progressivamente mudando de direção e ficando inclinado para direita. Quando o spinner perde o contato com a tampa, mais uma vez só atua a força peso, logo o seu torque em relação ao CM é nulo, acarretando a conservação do momento angular de spin numa direção inclinada e praticamente contida no plano xz, como mostra a Fig. 2.

Nesse momento, uma aparente contradição emerge: se as forças são idênticas em ambos os casos, por que os momentos angulares de spin durante o voo estão orientados para direções diferentes? Por que os torques aplicados pela tampa, em relação ao ponto fixo $\boldsymbol{O}$, têm direções distintas?

De fato, no caso anterior só havia uma componente do torque no sentido do eixo y, mas agora há duas componentes de torque: uma no sentido de y que dá origem ao momento angular orbital e outra no sentido $\mathbf{x}$, res- 
ponsável por inclinar para direita o momento angular de spin. Sabemos do caso 1 que a resultante entre a força normal e a componente da força peso ortogonal à tampa é responsável pela componente do torque no sentido y, mas que força provocaria uma componente do torque no sentido $\mathbf{x}$ ?

A resposta dessa última questão é a força normal. Quando o spinner está em repouso sobre a tampa, a força normal tem o mesmo módulo e direção da força peso, mas sentido oposto e também está aplicada sobre o CM. À medida que, a tampa se movimenta, ocorrem duas coisas com a força normal: ela aumenta de módulo e se desloca sobre a base do spinner no sentido do eixo y, mudando o seu ponto de aplicação do CM para um ponto da borda de sua base.

Qual a causa do deslocamento do ponto de aplicação da força normal? É um desequilíbrio dinâmico provocado pela rotação da tampa em torno do eixo y ao empurrar o spinner que já estava em rotação de spin em torno do eixo z. Realmente, no início, o spinner já possui um momento angular de spin na direção $\mathbf{z}$, mas como o torque resultante sobre ele aponta no sentido y, então ocorre uma variação do momento angular de spin no sentido do torque, forçando o spinner a virar para o lado de dentro da figura, ou melhor, girar em torno do eixo -x. Contudo, a base do spinner está sobre a tampa que bloqueia essa rotação através de um deslocamento da força de reação normal do CM para um ponto da borda da base.

Qual a consequência do deslocamento do ponto de aplicação da força normal? Essa mudança do ponto de aplicação da força normal altera levemente a direção do vetor posição, medido em relação à origem $\boldsymbol{O}$ e, por extensão, a direção torque resultante. Ao passo que, a tampa vai virando, pela regra da mão direita, o torque resultante sobre o spinner terá uma componente no sentido de $\mathbf{y}$, assim como outra componente no plano $\mathbf{x z}$, em uma direção inclinada em relação ao eixo x. Essa projeção do torque total no sentido $\mathbf{x}$ que não existia no caso 1 é responsável por inclinar o momento angular de spin para a direita. Essa inclinação do momento angular de spin corresponde a um ângulo de aproximadamente $40^{\circ} \mathrm{em}$ relação ao eixo $\mathbf{z}$, como pode ser inferido da videoanálise do caso 2 .

\subsection{Análise quantitativa dos resultados}

As Figs. 3 e 4 mostram os screenshots da tela do software livre Tracker exibindo as vidioanálises e os dados obtidos do CM e de um ponto da borda do spinner em ambos os lançamentos.

É possível visualizar a trajetória do CM e de um ponto da borda do spinner em ambos os casos. Observando as trajetórias a partir do referencial $\boldsymbol{O}$ não é possível discutir com clareza as diferenças e semelhanças em cada caso. Todas as trajetórias parecem ser parabólicas com concavidades levemente diferentes.

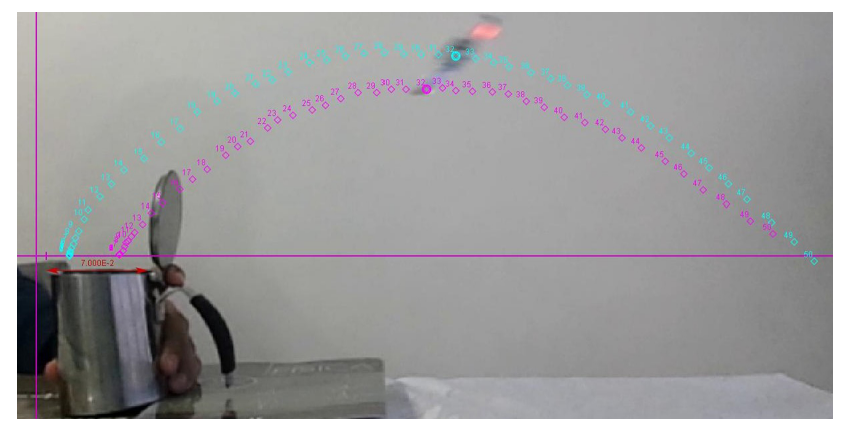

Figura 3: Screenshot da videoanálise do lançamento oblíquo do spinner sem rotação. São mostradas a videoanálise do movimento do centro de massa (CM) e de um ponto da sua borda.

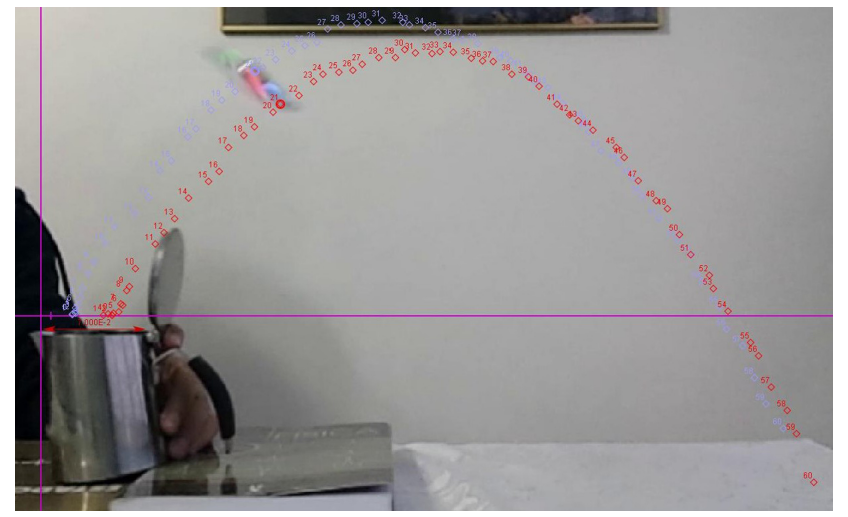

Figura 4: Screenshot da videoanálise do lançamento oblíquo do spinner com rotação. São mostradas a videoanálise do movimento do centro de massa (CM) e de um ponto da sua borda.

Com o objetivo de verificar se essas trajetórias são ou não parabólicas, foram analisadas a dependência da componente vertical (eixo $\mathbf{z}$ ) com o tempo. A Fig. 5 mostra o ajuste quadrático da componente $\mathbf{z}$ (vertical) para do CM do spinner no caso do lançamento oblíquo sem spin. É visível que o ajuste quadrático é bem razoável

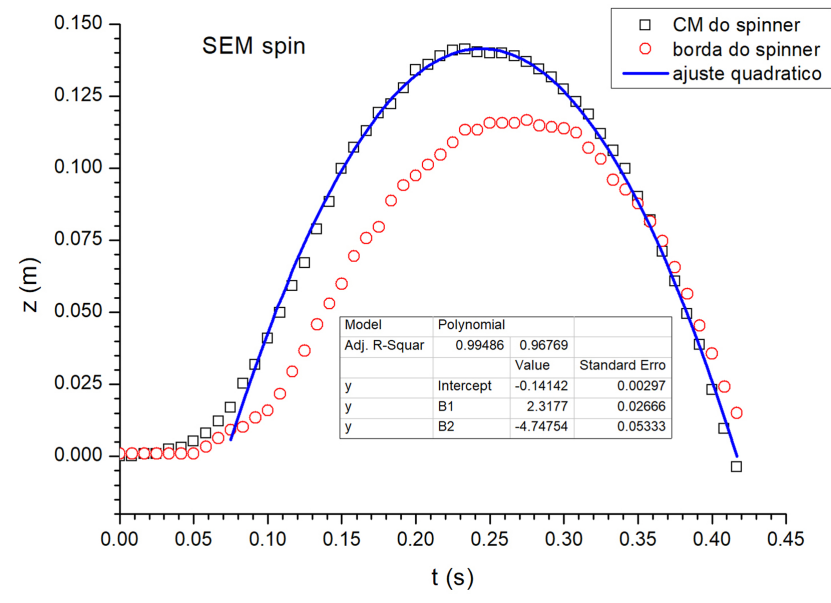

Figura 5: Ajuste quadrático da componente vertical (eixo z) em função do tempo para o $\mathrm{CM}$ e pontos experimentais da borda do spinner no caso do lançamento oblíquo do spinner sem rotação. 
para a componente $\mathbf{z}$ do $\mathrm{CM}\left(\mathrm{R}^{2}=0,995\right)$. O valor da aceleração fornecido pelo ajuste é de aproximadamente $9,5 \sqrt{1} \mathrm{~m} / \mathrm{s}^{2}$, compatível com o valor oficial da gravidade nessa latitude, dado por $\mathrm{g}=9,7877 \mathrm{~m} / \mathrm{s}^{2}[7]$.

A Fig. 6 mostra os ajustes quadráticos para a componente $\mathbf{z}$ (vertical) para o CM e um ponto da borda do spinner no caso do lançamento oblíquo com spin. É visível que o ajuste quadrático é novamente razoável para a componente $\mathbf{z}$ tanto para o $\mathrm{CM}$ como para a borda do spinner $\left(\mathrm{R}^{2}=0,995\right)$. O valor da aceleração fornecido pelo ajuste em ambos os casos é de aproximadamente $9,6 \sqrt{1} \mathrm{~m} / \mathrm{s}^{2}$, que é novamente compatível com o valor da aceleração da gravidade local, fornecendo assim uma pista de que o spinner mantém sua orientação durante o voo.

A fim de corroborar a conclusão anterior, utilizando os dados de posição e tempo, foi investigada a trajetória de um ponto da borda do spinner no plano $\mathbf{x z}$, tendo como

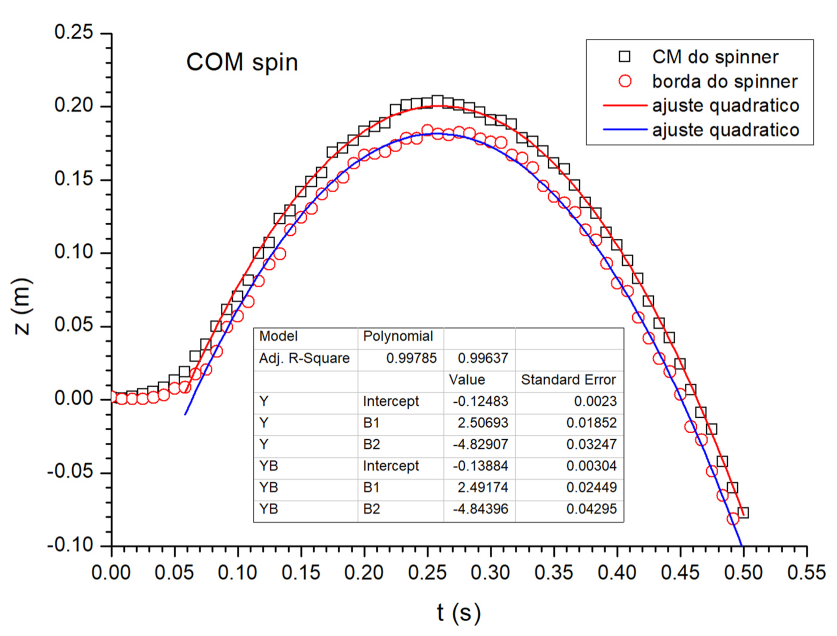

Figura 6: Ajuste quadrático da componente vertical (eixo z) em função do tempo para o CM e pontos experimentais da borda do spinner no caso do lançamento oblíquo do spinner com rotação.

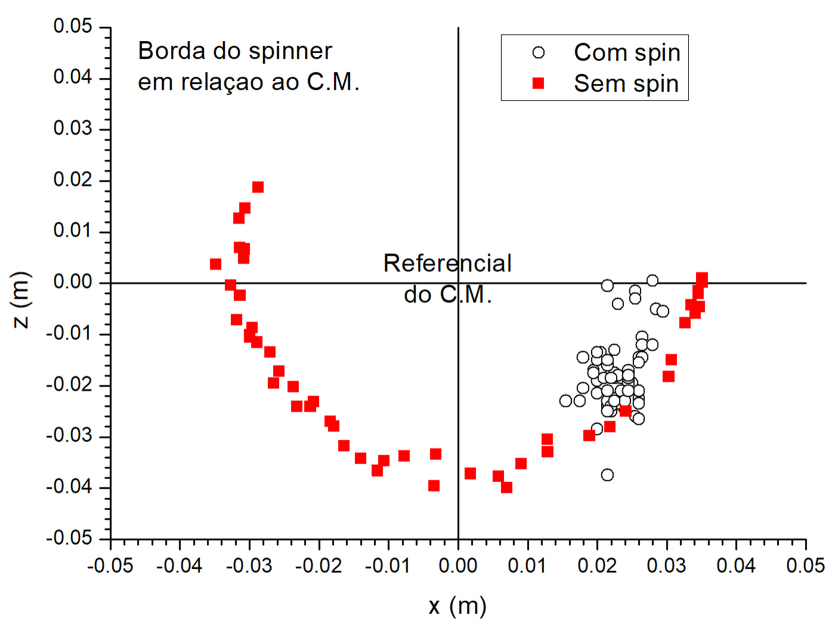

Figura 7: Trajetórias de um ponto da borda do spinner nos dois lançamentos oblíquos (com e sem rotação) vistos do referencial do $\mathrm{CM}$. referência o CM em cada caso, como mostra a Fig. 7. Observando-se os pontos circulares aglutinados no quarto quadrante, pode-se concluir que o spinner não gira em torno do CM quando é lançado com spin. Por outro lado, os pontos experimentais mostram que a trajetória do ponto da borda do spinner é circular, evidenciando seu giro em torno do CM quando é lançado sem spin.

A referência [4] determina os momentos de inércia de um spinner de massa $M=61 \mathrm{~g}$, em relação aos seus eixos principais de simetria. Supondo que o spinner utilizado neste experimento tenha uma distribuição de massa similar, e considerando que sua massa é $29 \mathrm{~g}$, podemos estimar que os momentos de inércia do spinner utilizados neste artigo sejam aproximadamente metade daqueles obtidos em [4]:

$$
I_{y^{\prime}} \approx 8,0 \times 10^{-6} \mathrm{~kg} \cdot \mathrm{m}^{2}
$$

$$
I_{z^{\prime}} \approx 1,7 \times 10^{-5} \mathrm{~kg} \cdot \mathrm{m}^{2}
$$

No caso 1, o eixo principal de simetria y' coincide com o eixo y do sistema de coordenadas fixo. Através da videoanálise da rotação do spinner em torno do seu CM nesse caso, podemos estimar a velocidade angular em torno do CM no sentido y', em:

$$
\omega_{y^{\prime}}=8,6 \mathrm{rad} / \mathrm{s}
$$

Por sua vez, no caso 2, o eixo principal de simetria $\mathbf{z}^{\prime}$ coincide com o eixo $\mathbf{z}$ do sistema de coordenadas fixo, apenas no instante inicial quando o spinner ainda está na posição horizontal, sobre a tampa. A videoanálise do movimento do spinner nessa circunstância, antes do lançamento oblíquo, fornece a seguinte estimativa para a velocidade angular:

$$
\omega_{z^{\prime}} \approx 85 \mathrm{rad} / \mathrm{s}
$$

Utilizando os valores obtidos em (3-6) podemos estimar os valores das componentes de momento angular de spin:

$$
\begin{aligned}
& S_{y^{\prime}} \approx 6,8 \times 10^{-5} \mathrm{~kg} \cdot \mathrm{m}^{2} / \mathrm{s}(\text { caso } 1) \\
& S_{z^{\prime}} \approx 1,4 \times 10^{-3} \mathrm{~kg} \cdot \mathrm{m}^{2} / \mathrm{s}(\text { caso } 2)
\end{aligned}
$$

Observe que a razão dos momentos de inércia é $I_{z^{\prime}} / I_{y^{\prime}} \approx$ 2 , enquanto a razão das velocidades angulares é dada por $\omega_{z^{\prime}} / \omega_{y^{\prime}} \approx 10$. Isso nos leva a seguinte razão para as componentes dos momentos angulares de spin:

$$
\frac{S_{z^{\prime}}}{S_{y^{\prime}}} \approx 20
$$

Portanto, podemos concluir que o momento angular de spin no caso 2 é cerca de 20 vezes maior em magnitude do que o momento angular de spin no caso 1. 


\section{Conclusões}

Os livros consagrados para o ensino de física básica em cursos superiores costumam definir os conceitos de torque e de momento angular primeiramente para uma partícula $[8,9]$. Depois, definem o momento de inércia e o respectivo momento angular de um corpo rígido em torno de um eixo fixo, como um caso particular do momento angular de um sistema de partículas. Por fim, são apresentados exemplos de situações reais que envolvam esses conceitos, tais como uma esfera ou cilindro rolando sem deslizar em uma mesa ou em um plano inclinado, um giroscópio e/ou um ioiô. Infelizmente, em todos esses exemplos, o momento angular orbital do $\mathrm{CM}$ em relação a um ponto fixo é ignorado e apenas se discute a relação entre o torque e o momento angular de spin, em relação ao CM.

Neste trabalho uma situação real composta por duas configurações experimentais extremamente simples, que pode ser utilizada em sala de aula para promover o aprendizado dos conceitos de torque, momento angular orbital e momento angular de spin, utilizando um famoso brinquedo que atrai a atenção e a curiosidade dos estudantes

Sem dúvida o resultado mais importante foi mostrar que é possível, através da videoanálise do lançamento oblíquo de um fidget spinner, abordar de forma qualitativa e simultânea os conceitos de torque, momento angular orbital e momento angular de spin. Além disso, a videoanálise também possibilita uma estimativa quantitativa das magnitudes dos momentos angulares de spin em relação ao respectivo eixo principal de simetria do spinner, em ambos os casos.

\section{Agradecimentos}

Gostaríamos de agradecer ao estudante José Vitor Mendes de Jesus pela ajuda durante a filmagem dos lançamentos oblíquos do spinner.

\section{Referências}

[1] D. Macisaac, The Physics Teacher 55, 384 (2017).

[2] D.U. Suwarno, Journal of Science and Science Education 1, 75 (2017)

[3] J. Marks e P. Bradfield, Physics World 30, 20 (2017).

[4] V.L.B. de Jesus e D.G G. Sasaki, The Physics Teacher 56, 639 (2018).

[5] http://physlets.org/tracker/, acessado em $22 / 08 / 2019$.

[6] S.T. Thornton e J.B. Marion, Classical Dynamics of Particles and System (Brooks/Cole, Belmont, 2004) $5^{\mathrm{a}} \mathrm{ed.}$

[7] V.L.B. de Jesus, Experimentos e Videoanálise: Dinâmica (Livraria da Física, São Paulo, 2014).

[8] P.A. Tipler e G. Mosca, Física para Cientistas e Engenheiros (LTC Livros Técnicos e Científicos, Rio de Janeiro, 2009), $6^{\mathrm{a}}$ ed.

[9] D. Halliday, R. Resnick e J. Walker, Fundamentos de Física, (LTC Livros Técnicos e Científicos, Rio de Janeiro, 2009). 